\title{
Royal Observatory, Greenwich
}

\section{Annual Visitation}

$\mathrm{T}$ HE Astronomer Royal read his annual report to the Board of Visitors of the Royal Observatory, Greenwich, on June 6 last. Although the weather was far from ideal, the Garden Party which is associated with the visitation was well attended, and visitors and guests were shown the new Reversible Transit Circle referred to in the Report. The erection of this instrument was commenced in February and completed in March, but actual observations have not yet been made with it, as it was discovered by tests that the pivots were slightly elliptical in shape ; in the meantime, work has been done on the pivots which it is hoped will shortly be circular within the required limits. This Transit Circle is in the Christie Enclosure, near the Yapp Reflector, and is accordingly some little distance east of the adopted Greenwich Meridian on which Airy's Transit Circle will continue to stand; the new instrument is reversible and has a travelling micrometer wire which is driven by an electric motor at the speed appropriate to the declination of the star under observation. It is intended to secure observations in fundamental astronomy which will be more accurate than those obtainable with the Airy's Transit Circle, and we may await with some interest the appearance of the results obtained with the new instrument.

A new Free Pendulum Clock is being presented to the Observatory by Mr. H. R. Fry. This clock is a Shortt clock which is similar to those at present in use, except that the slave pendulum of the new clock is of a much more refined construction than the standard pattern and is fully jewelled throughout. The time will be taken from the new clock from the free pendulum itself using a photo-electric method.

The routine astronomical work of the Observatory continues. During the year, 9,899 transit observations and 9,409 observations of zenith distance were secured. Attention has been directed to the minor planets, which are being observed with the intention of using them to fix the equinox and equator point, and 42 transit observations of minor planets were secured during the year, in addition to 139 photographic observations secured with the Astrographic Equatorial. The Astronomer Royal directs special attention to the successful application to the measurement of line contour with the spectrohelioscope which has recently been accomplished at Greenwich. A programme of photometric observations of intensity of bright eruptions and dark markings on the solar disk is now in hand. The observers use a visual method of photometry, and the results promise to be of interest in connexion with the investigation of conditions in the ionosphere besides having astrophysical significance.

The mean values of the magnetic elements at Abinger in 1935 are given below, together with values in recent years for comparison:

$\begin{array}{ccllr} & \text { Declination, W } & \begin{array}{c}\text { Horizontal } \\ \text { Intensity }\end{array} & \begin{array}{c}\text { Vertical } \\ \text { Intensity }\end{array} & \text { Inclination } \\ 1932 & 12^{\circ} 2 \cdot 6^{\prime} & 0 \cdot 18536 & 0 \cdot 42940 & 66^{\circ} 39 \cdot 1^{\prime} \\ 1933 & 11^{\circ} 51 \cdot 7^{\prime} & 0 \cdot 18532 & 0 \cdot 42942 & 66^{\circ} 39 \cdot 4^{\prime} \\ 1934 & 11^{\circ} 41 \cdot 1^{\prime} & 0 \cdot 18533 & 0 \cdot 42955 & 66^{\circ} 39 \cdot 7^{\prime} \\ 1935 & 11^{\circ} 30 \cdot 3^{\prime} & 0 \cdot 18527 & 0.42981 & 66^{\circ} 40 \cdot 9^{\prime}\end{array}$

Regular observations of the amount of solid matter polluting the atmosphere have been continued. A comparison between the pollution at Greenwich during the winter of $1934-35$ with the pollution at the worst London station (Westminster) and the worst Glasgow station reveal the fact that Greenwich experiences rather more than fifty per cent more pollution than either of the other stations. During the winter 1935-36, less pollution was observed at Greenwich : the decrease is attributed to the smaller proportion of wind coming from a northerly direction experienced in 1935-36, as the main source of pollution lies in the factory area along the Thames and the densely populated area north of the river, opposite Greenwich. The worst pollution occurs at $20^{\mathrm{h}}$, indicating domestic fires as an important source of atmospheric impurity.

The mean temperature for the twelve months ending April 30, 1936, was $50.2^{\circ}$, which is $0.7^{\circ}$ higher than the average for the seventy-five years 1841-1915. The year was remarkable for a temperature below freezing point registered on May 17, 1935.

Hitherto the time service maintained by the Observatory has suffered from a lack of precision in so far as the absolute personal equations of the observers who observe clock corrections with the Small Reversible Transit telescope are not known. A personal equation machine has been constructed with the view of determining these personalities. The machine carrying an artificial star has been installed on the roof of the Octagon Room, and the movement of this artificial star will be observed with a telescope similar to that used in the observations for clock correction which will be mounted on the roof of the Main Building. It is expected to commence these observations shortly. Meanwhile, the annual mean differences between the Greenwich time signals and those sent out by Paris, Nauen and Bordeaux are satisfactorily small, being $-0.009^{8}$, $-0.010^{\mathrm{s}}$ and $-0.009^{\mathrm{s}}$ respectively, the other ob. servatories being in the mean early on Greenwich.

The old system of employing unestablished com. puters at Greenwich is to go. This scheme was instituted by Sir George Airy, and it worked admirably in the past. Some of the boys employed as temporary computers were incorporated in the established steff and have gone on to render distinguished service as assistants at the Observatory ; others who have left have found their Greenwich training of great service to them, and several have gone on to hold very responsible appointments. But the times have changed and there is no longer a market amongst employers for an accomplished computer, with the result that computing at Greenwich is now a blindalley occupation for a young man, since all the com. puters could not be absorbed on the established staff. The Lords Commissioners of H.M. Treasury have approved a new scheme according to which the unestablished computers will disappear while the permanent staff will be increased, the computing duties being performed for the most part by twelve established women writing assistants. The transition will be made gradually. 